\title{
GENETICS
}

\section{New mutations in Cushing disease identified}

Pituitary adenomas from patients with Cushing disease have been shown to have somatic mutations in USP8, which encodes a deubiquitinase from the ubiquitin-specific family of proteases.

"So far the underlying genetic events in sporadic Cushing disease have remained obscure," explain Martin Reincke and Martin Fassnacht, two of the authors of the study. In an attempt to elucidate the mutations present in Cushing disease, the researchers performed exome sequencing on 10 paired samples of pituitary adenomas secreting adrenocorticotropic hormone and normal tissue from patients with Cushing disease.

Of these samples, four of the pituitary adenomas had somatic mutations in USP8; the mutations were not present in any of the normal tissue samples. The mutations were all found in the 14-3-3 protein binding motif and enhanced proteolytic cleavage and the catalytic activity of USP8. As a result, deubiquitination of epidermal growth factor receptor (EGFR) was increased, which impaired its downregulation and sustained EGFR signalling. High levels of EGFR stimulated transcription of POMC, which resulted in increased levels of adrenocorticotropic hormone and thus tumorigenesis.

The authors suggest that overexpression of USP8 and high levels of EGFR could be used as a diagnostic marker in these patients. These findings could also open up new therapeutic options. "The EGFR pathway is a wellestablished therapeutic target for other cancers and many EGFR-targeting drugs are available," say Reincke and Fassnacht. However, whether these drugs will work in patients with Cushing disease will need to be determined.

\section{Claire Greenhill}

Original article Reincke, M. et al. Mutations in the deubiquitinase gene USP8 cause Cushing's disease. Nat. Genet. doi:10.1038/ng.3166 